\title{
Contextualisation des pratiques et des recherches en éducation relative à l'environnement - Ancrage territorial et culturel
}

\section{Marianne von Frenckell}

\section{(2) OpenEdition}

\section{Journals}

Édition électronique

URL : http://journals.openedition.org/ere/4164

DOI : $10.4000 /$ ere.4164

ISSN : 2561-2271

Éditeur

Centr'ERE

Référence électronique

Marianne von Frenckell, « Contextualisation des pratiques et des recherches en éducation relative à

l'environnement - Ancrage territorial et culturel », Éducation relative à l'environnement [En ligne], Volume

5 | 2005, mis en ligne le 20 novembre 2005, consulté le 21 février 2020. URL : http://

journals.openedition.org/ere/4164; DOI : 10.4000/ere.4164 


\title{
Contextualisation des pratiques et des recherches en éducation relative à l'environnement - Ancrage territorial et culturel
}

\author{
Marianne von Frenckell
}

1 Dans une perspective d'éducation relative à l'environnement (ERE) qui se préoccupe de la construction du réseau des relations personne - groupe social - environnement et s'intéresse à des questions relatives au développement des personnes et des groupes sociaux en relation avec leur milieu de vie (Sauvé, 1997), la notion de contexte, inhérente à toute situation éducative, prend une dimension particulière. Le milieu de vie est partie intégrante du contexte d'apprentissage: les représentations de l'environnement s'y construisent, évoluent en lien dialectique avec l'agir dans et sur cet environnement. La dimension contextuelle est centrale dans toute démarche pédagogique qui vise à développer chez les personnes des savoir-être et des savoir-agir éclairés et responsables, en cohérence avec leurs choix de vie et de société. Les implications de cette dimension demandent à être explorées tant en ce qui concerne les pratiques éducatives singulières que le cadre dans lequel se développe l'ERE.

2 Quel est le milieu de vie au sein duquel se construit la relation environnementale ? Quel est le milieu de vie de référence (famille, quartier, village, ville où se situe mon école et où travaillent mes parents, cadre professionnel, lieux de loisirs, etc.) sachant que l'être humain, particulièrement dans les pays industrialisés, relève de systèmes d'appartenance multiples dont le commun dénominateur n'est pas nécessairement la proximité ? Dans quelle(s) « réalité(s) » s'ancrent les situations éducatives ? Quel est le contexte dans lequel elles sont signifiantes pour les acteurs qui y sont associés?

C'est au niveau local que «la participation active des individus et groupes sociaux à la solution des problèmes environnementaux" et les opportunités de "donner aux apprenants la possibilité de prendre des décisions » (UNESCO-PNUE, 1978) prônées dans les textes fondateurs de l'ERE peuvent prendre sens et que se construisent des attitudes 
relatives au "pouvoir agir ». Les enjeux environnementaux y sont ancrés dans le vécu des groupes et des personnes, socialement construits en fonction des contextes biophysiques, sociaux, politiques, culturels, historiques, etc. L'action collective s'y expérimente à travers des interventions significatives pour la compréhension des réalités quotidiennes des acteurs. Diverses expériences de contribution à l'éducation relative à l'environnement menées au sein de communautés d'apprentissage mettent en évidence la dynamique des processus d'engagement des personnes et des groupes sociaux envers leur milieu de vie.

Par les approches pédagogiques qu'elle privilégie, par les dynamiques qu'elle stimule, et par l'expérimentation et l'enracinement de ses processus dans la réalité même, la communauté d'apprentissage apparaît particulièrement appropriée pour la découverte du milieu de vie, l'identification et la compréhension des interrelations, l'analyse et l'étude des contextes et problématiques socioenvironnementales, la recherche de solutions, ainsi que le développement de la créativité et de l'imaginaire pour mettre en œuvre de nouveaux projets sociaux. (Orellana, 2004, p. 67)

Cependant, ce qu'il est convenu d'appeler la crise environnementale est planétaire et les individus et groupes sociaux sont de plus en plus interdépendants à l'échelle globale. Toute action locale peut avoir un effet global et les problèmes globaux, largement diffusés par les médias, ne manquent pas d'influencer les visions locales. Quels sont les éléments de contexte à mobiliser dans cette perspective d'une éducation que Beck (1999) appelle " g-locale » sans verser dans la pensée totalisante qui, trop souvent, accompagne la prise en compte de la planète dans sa globalité ? Contrairement aux problématiques environnementales construites par les acteurs locaux, les problèmes environnementaux globaux sont définis par les "experts » et souvent répercutés par les médias avec une connotation catastrophiste. En général, les populations ne sentent pas de prise directe sur ces problèmes définis sans elles et loin d'elles; elles n'en appréhendent pas les éventuelles relations avec leur réalité immédiate ; ils ne sont pas porteurs d'un « pouvoir agir ici et maintenant». De plus, le langage de la «durabilité » envahit le discours environnemental ; la logique marchande propose de " gérer » notre planète, centrant sur « l'utilitaire » la relation Être Humain Nature au nom du concept obligé de développement durable.

5 Face (en réaction?) au discours environnemental globalisant et à la mondialisation de l'économie tendant à imposer un modèle unique de développement, il est surprenant de constater à quel point les singularités territoriales reviennent en force au niveau institutionnel à travers les démarches de décentralisation et d'autonomisation. C'est, en effet, à l'échelle des territoires que les problèmes socio-environnementaux sont perçus; cette notion de territoire qui conjugue des représentations individuelles et collectives, sociales et culturelles, des dimensions identitaire, symbolique, politique, historique, etc. définit en quelque sorte le contexte où l'individu peut agir en tant que citoyen.

[...] le territoire témoigne d'une appropriation à la fois économique, idéologique et politique (sociale donc) de l'espace par des groupes qui se donnent une représentation particulière d'eux-mêmes, de leur histoire, de leur singularité. [...] Le territoire c'est d'abord une idée, la volonté d'un groupe social de tisser du lien social localisé, de créer des solidarités à l'échelle locale, de se vouloir co-auteur d'un développement de proximité. [Il se trouve] au point de rencontre de la nature et de la culture, des sociétés et de leur environnement, mais aussi dans le champ de la durée et de l'histoire, [...]. [Le territoire concrétise] la façon dont l'Homme et ses 
sociétés se représentent, conçoivent et produisent leurs rapports à l'espace [...]. (Di Meo, 1998)

Comme le fait remarquer Jacques Theys (2002) « [...] c'est à l'échelle des territoires que le «développement durable » a été le plus rapidement et visiblement intégré dans les politiques - et ceci sous les formes les plus diverses - : Agendas 21, [...]». Ces Agendas 21 locaux et autres plans locaux de développement durable favorisent-ils l'émergence d'une citoyenneté "g-locale», offrent-ils un lieu où sont questionnées, transformées les relations des individus et des groupes sociaux avec leur milieu de vie? La suite de l'analyse de Jacques Theys fournit des éléments de réponse :

Il y a, en effet, de bonnes raisons de penser [...] que c'est essentiellement à l'échelle des territoires que pourront être construites, démocratiquement, les articulations indispensables entre les dimensions sociales et écologiques du développement durable.[...].

On est [...] en droit de se demander si, sous couvert d'intentions louables, se référant aux générations futures, une élite technocratique, armée d'un discours moralisateur sur l'état de la planète et les responsabilités de chacun, n'est pas en train d'imposer une nouvelle vision du monde, un «nouvel évangile» auquel personne n'aurait explicitement souscrit. Nombre de mesures proposées, qui visent à modifier les comportements quotidiens, les types de consommation, les modes de déplacement, s'appuient en effet sur des principes ou des normes dont la légitimité n'est pas acquise et parfois sur des valeurs faussement partagées en dehors d'un cercle d'experts. [...] C'est dans un tel contexte que se justifient tout particulièrement les tentatives d'ouverture démocratique mises en œuvre essentiellement à l'échelle des territoires. [...] il faut bien remarquer que beaucoup des expériences ou actions engagées en restent souvent à l'état de réflexion, de l'effet d'annonce ou de l'affichage, sauf exception [...] une certaine confusion est entretenue sur la contribution réelle de ces projets à la solution des problèmes globaux. Entre le local et le global les articulations restent encore largement à construire. (Theys, 2002)

7 Force est, en effet, de constater que même si de nombreux Agendas 21 mettent en avant l'implication communautaire et la construction participative des projets, l'intégration d'une ERE visant à changer la façon dont les acteurs pensent le monde et se pensent en relation avec lui n'est guère au cœur de la mise en œuvre de nombreux d'entre eux. Sans stratégie éducative appropriée, ces projets, pourtant développés dans des contextes territoriaux potentiellement riches de lien social ne semblent pas générer un véritable processus de changement individuel et collectif.

Envahissant le discours environnemental, le langage de la durabilité se fait aussi de plus en plus présent dans le champ de l'ERE. Pour certains, l'ERE ne serait qu'une facette de l'éducation pour le développement durable. De nombreuses critiques ont déjà été émises quant aux dérives idéologiques possibles (entre autres : Jickling, 1994 ; Sauvé, 1996). Le problème de l'articulation entre le local et le global nous semble également devoir être souligné : les solutions de continuité restent à construire; à défaut, la discordance entre la finalité de "durabilité " annoncée et les niveaux d'engagement individuel sera génératrice de sentiments d'impuissance. Enfin, quels savoir-agir peuvent se développer face à des problèmes environnementaux construits par d'autres dans un contexte culturel souvent éloigné de celui des acteurs engagés dans des actions collectives locales?

9 Les questions ne manquent donc pas concernant les contextes territoriaux dans lesquels se mènent les interventions en ERE et plus particulièrement concernant leurs liens avec les dimensions naturelle, sociale, culturelle, historique, etc. dont ils sont 
porteurs. Ce volume 5 de la revue Éducation relative à l'environnement: Regards Recherches - Réflexions veut participer à ce questionnement. Le thème de ce volume "Cultures et territoires: ancrages pour une éducation relative à l'environnement " propose aux auteurs de clarifier, dans une perspective critique, les notions d'ancrage territorial et d'ancrage culturel de l'ERE; d'explorer les relations entre les modes d'intervention, d'apprentissage et de construction des savoirs et leurs contextes d'émergence; d'analyser les liens entre l'enracinement territorial des pratiques et la dimension culturelle de l'ERE; de mettre en évidence les enjeux pédagogiques liés à la prise en compte des contextes d'apprentissage d'un savoir-vivre ensemble solidaire et responsable.

10 Comme dans les volumes précédents, les Regards, Recherches et Réflexions sont pluriels. Les origines géographiques (Afrique, Amérique du Nord, Amérique Centrale, Europe) et les réalités (urbaines, rurales, de montagne) sont multiples. Si elles sont loin de cerner la question, les différentes contributions donnent à voir la façon dont les auteurs appréhendent cet ancrage de l'ERE dans les territoires et cultures et mettent en évidence la diversité tant des pratiques de terrain et que des pratiques de recherche. Nous espérons qu'elles contribueront à alimenter le questionnement et seront la base de discussions fructueuses.

\section{BIBLIOGRAPHIE}

Beck, U. (1999). World risk society. Frankfurt a.M. : Suhrkamp.

Di Meo, G. (1998). Géographie sociale et territoriale. Paris : Nathan Université.

Jickling, B. (1994). Studying sustainable development. Problems and possibilities. Canadian Journal of Education, 19, 232-240.

Orellana, I. (2005). L'émergence de la communauté d'apprentissage ou l'acte de recréer des relations dialogiques et dialectiques de transformation du rapport au milieu de vie. In Sauvé, L., Orellana, I. et van Steenberghe, E. (dir.), Éducation et environnement - Un croisement de savoirs, Actes $\mathrm{du}$ colloque « Le croisement des savoirs au cœur des recherches en éducation relative à l'environnement » dans le cadre du 72e Congrès de l'Acfas (p. 67-68). Montréal : Éditions Fides, Collection «Cahiers scientifiques de l’Acfas », nº 104.

Sauvé, L. (1996). Environmental education and sustainable development : Further appraisal. Canadian Journal of Environmental Education, 1, 7-34.

Sauvé, L. (1997). Pour une éducation relative à l'environnement (2e édition). Montréal : Guérin. Theys, J. (2002). L'approche territoriale du « développement durable », condition d'une prise en compte de sa dimension sociale. Revue Développement durable et territoires, Dossier 1 : Approches territoriales du développement durable. Consulté le 30 novembre 2005 sur http:// developpementdurable.revues.org/document1475.html. 
UNESCO-PNUE. (1978). Conférence intergouvernementale sur l'éducation relative à l'environnement, Tbilissi, 14-26 octobre 1977. Paris : UNESCO.

\section{AUTEUR}

MARIANNE VON FRENCKELL

Université de Liège, Belgique 